Pacific Journal of Mathematics

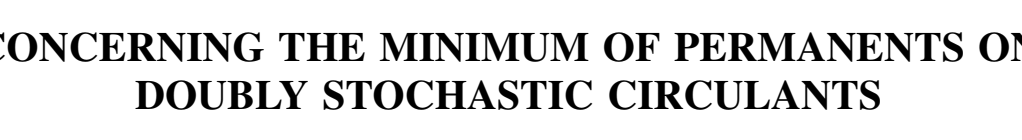




\title{
CONCERNING THE MINIMUM OF PERMANENTS ON DOUBLY STOCHASTIC CIRCULANTS
}

\author{
Gerald E. Suchan
}

Let $P_{n}$ be the permutation matrix such that $\left(P_{n}\right)_{i j}=1$ if $j=i+1(\bmod n)$. Minc $[2]$ proved that the minimum of the permanent on the collection of $n \times n$ doubly stochastic circulants $\alpha I_{n}+\beta P_{n}+\gamma P_{n}^{2}$ is in $\left(1 / 2^{n}, 1 / 2^{n-1}\right]$, and if $n \geqq 5$ then the minimum is not achieved at $(1 / 3) I_{n}+(1 / 3) P_{n}+(1 / 3) P_{n}^{2}$. This paper proves that if $n \geqq 3$ then the minimum of such permanents is less than $1 / 2^{n-1}$, and if $n \in\{3,4\}$ then this minimum is uniquely achieved at $(1 / 3) I_{n}+(1 / 3) P_{n}+(1 / 3) P_{n}^{2}$.

Introduction. Let $n$ be a positive integer, let $I_{n}$ denote the $n \times n$ identity matrix, and let $P_{n}$ denote the full cycle permutation matrix such that $\left(P_{n}\right)_{i j}=1$ if $j=i+1(\bmod n)$. Minc [2] studied the permanent of circulants $\alpha I_{n}+\beta P_{n}+\gamma P_{n}^{2}$ and proved the following three theorems:

THEOREM 1. If $n \geqq 3$ then

$$
\begin{aligned}
\operatorname{per}\left(\alpha I_{n}+\beta P_{n}+\gamma P_{n}^{2}\right)= & \left(\frac{\beta+\sqrt{\beta^{2}+4 \alpha \gamma}}{2}\right)^{n} \\
& +\left(\frac{\beta-\sqrt{\beta^{2}+4 \alpha \gamma}}{2}\right)^{n}+\alpha^{n}+\gamma^{n}
\end{aligned}
$$

THEOREM 2. If $\alpha, \beta, \gamma$ are nonnegative then

$$
\frac{1}{2^{n}}<\min _{\alpha+\beta+\gamma=1} \operatorname{per}\left(\alpha I_{n}+\beta P_{n}+\gamma P_{n}^{2}\right) \leqq \frac{1}{2^{n-1}} .
$$

THEOREM 3. If $\alpha, \beta, \gamma$ are nonnegative, $n \geqq 5$, then

$$
\min _{\alpha+\beta+\gamma=1} \operatorname{per}\left(\alpha I_{n}+\beta P_{n}+\gamma P_{n}^{2}\right)<\operatorname{per}\left(\frac{1}{3} I_{n}+\frac{1}{3} P_{n}+\frac{1}{3} P_{n}^{2}\right) \text {. }
$$

MAIN Results. Let $S=\{(\alpha, \gamma) \mid 0 \leqq \alpha, 0 \leqq \gamma, \alpha+\gamma \leqq 1\}$, and let $f_{n}$ denote the function on $S$ such that

$$
f_{n}(\alpha, \gamma)=\operatorname{per}\left(\alpha I_{n}+(1-\alpha-\gamma) P_{n}+\gamma P_{n}^{2}\right) .
$$

THEOREM 4. If $n \geqq 3$ then $f_{n}$ is not minimum on the boundary of $S$.

Lemma to Theorem 4. The minimum of $f_{n}$ on the boundary of 
$S$ is $1 / 2^{n-1}$. If $n$ is even this minimum is achieved only on $\{(1 / 2,0),(0,1 / 2)\}$, and if $n>1$ and $n$ is odd this minimum is achieved only on $\{(1 / 2,0),(1 / 2,1 / 2),(0,1 / 2)\}$.

Proof. The lemma is clearly true is case $n \in\{1,2\}$. Suppose $n \geqq 3$. Since

$$
f_{n}(1 / 2,0)=f_{n}(0,1 / 2)=\frac{1}{2^{n-1}}<1=f_{n}(1,0)=f_{n}(0,0) f_{n}(0,1),
$$

then it is sufficient to consider only points belonging to the interior of the boundary of $S$. The only real number $\alpha$ satisfying $D_{1} f_{n}(\alpha, 0)=0$ is $1 / 2$. Therefore, since $f_{n}(\alpha, \gamma)=f_{n}(\gamma, \alpha)$, then the minimum of $f_{x}$ on $\{(\alpha, \gamma) \mid \alpha \gamma=0\}$ is $1 / 2^{n-1}$. Let $g(\alpha)=f_{n}(\alpha, 1-\alpha)$. If $n$ is even, put $k=n / 2$ and observe that $g(\alpha)=\left(\alpha^{k}+(1-\alpha)^{k}\right)^{2}$. If $n$ is odd then $g(\alpha)=\alpha^{n}+(1-\alpha)^{n}$. In either case, $1 / 2$ is the only real number $\alpha$ such that $g^{\prime}(\alpha)=0$. If $n$ is even then $f_{n}(1 / 2,1 / 2)=$ $1 / 2^{n-2}>1 / 2^{n-1}$, and if $n$ is odd then $f_{n}(1 / 2,1 / 2)=1 / 2^{n-1}$.

Proof of Theorem 4. By the lemma it is sufficient to show there is a point $q$ of $S$ so that $f_{n}(q)<f_{n}(1 / 2,0)$. Observe that $D_{1} f_{n}(\alpha, \gamma)$ is

$$
\begin{aligned}
& \frac{n}{2}\left(\frac{1-\alpha-\gamma+\sqrt{(1-\alpha-\gamma)^{2}+4 \alpha \gamma}}{2}\right)^{n-1}\left(-1+\frac{-1+\alpha+3 \gamma}{\sqrt{(1-\alpha-\gamma)^{2}+4 \alpha \gamma}}\right) \\
& \quad+\frac{n}{2}\left(\frac{1-\alpha-\gamma-\sqrt{(1-\alpha-\gamma)^{2}+4 \alpha \gamma}}{2}\right)^{n-1}\left(-1-\frac{-1+\alpha+3 \gamma}{\sqrt{(1-\alpha-\gamma)^{2}+4 \alpha \gamma}}\right) \\
& \quad+n \alpha^{n-1} .
\end{aligned}
$$

Thus $D_{1} f_{n}(1 / 2,0)=0$ and therefore, since $D_{1} f_{n}(\alpha, \gamma)=D_{2} f_{n}(\gamma, \alpha)$, then $(1 / 2,0)$ is a critical point for $f_{n}$. Now observe that $D_{1,1}(\alpha, \gamma)$ is

$$
\begin{aligned}
& \frac{n}{2}\left[\frac{(n-1)}{2}\left(\frac{1-\alpha-\gamma+\sqrt{(1-\alpha-\gamma)^{2}+4 \alpha \gamma}}{2}\right)^{n-2}\left(-1+\frac{-1+\alpha+3 \gamma}{\sqrt{(1-\alpha-\gamma)^{2}+4 \alpha \gamma}}\right)^{2}\right. \\
& \left.+\left(\frac{1-\alpha-\gamma+\sqrt{(1-\alpha-\gamma)^{2}+4 \alpha \gamma}}{2}\right)^{n-1}\left(\frac{(1-\alpha-\gamma)^{2}+4 \alpha \gamma-(-1+\alpha+3 \gamma)^{2}}{\left((1-\alpha-\gamma)^{2}+4 \alpha \gamma\right)^{3 / 2}}\right)\right] \\
& +\frac{n}{2}\left[\frac{(n-1)}{2}\left(\frac{1-\alpha-\gamma-\sqrt{(1-\alpha-\gamma)^{2}+4 \alpha \gamma}}{2}\right)^{n-2}\left(-1-\frac{-1+\alpha+3 \gamma}{\sqrt{(1-\alpha-\gamma)^{2}+4 \alpha \gamma}}\right)^{2}\right. \\
& \left.+\left(\frac{1-\alpha-\gamma-\sqrt{(1-\alpha-\gamma)^{2}+4 \alpha \gamma}}{2}\right)^{n-1}\left(\frac{-(1-\alpha-\gamma)^{2}+4 \alpha \gamma+(-1+\alpha+3 \gamma)^{2}}{\left((1-\alpha-\gamma)^{2}+4 \alpha \gamma\right)^{3 / 2}}\right)\right] \\
& +n(n-1) \alpha^{n-2} \cdot
\end{aligned}
$$

Thus $D_{1,1} f_{n}(1 / 2,0)=n(n-1) / 2^{n-3}$, and since $D_{2,2} f_{n}(\alpha, \gamma)=D_{1,1}(\gamma, \alpha)$ then $D_{2,2} f_{n}(1 / 2,0)=0$. Finally, observe that $D_{1,2} f_{n}(\alpha, \gamma)$ is 


$$
\begin{aligned}
\frac{n}{2} & {\left[\frac{(n-1)}{2}\left(\frac{1-\alpha-\gamma+\sqrt{(1-\alpha-\gamma)^{2}+4 \alpha \gamma}}{2}\right)^{n-2}\left(-1+\frac{-1+3 \alpha+\gamma}{\sqrt{(1-\alpha-\gamma)^{2}+4 \alpha \gamma}}\right)\right.} \\
& \times\left(-1+\frac{-1+\alpha+3 \gamma}{\sqrt{(1-\alpha-\gamma)^{2}+4 \alpha \gamma}}\right)+\left(\frac{1-\alpha-\gamma+\sqrt{(1-\alpha-\gamma)^{2}+4 \alpha \gamma}}{2}\right)^{n-1} \\
& \left.\times\left(\frac{3\left((1-\alpha-\gamma)^{2}+4 \alpha \gamma\right)-(-1+\alpha+3 \gamma)(-1+3 \alpha+\gamma)}{\left((1-\alpha-\gamma)^{2}+4 \alpha \gamma\right)^{3 / 2}}\right)\right] \\
+ & \frac{n}{2}\left[\frac{(n-1)}{2}\left(\frac{1-\alpha-\gamma-\sqrt{(1-\alpha-\gamma)^{2}+4 \alpha \gamma}}{2}\right)^{n-2}\left(-1-\frac{-1+3 \alpha+\gamma}{\sqrt{(1-\alpha-\gamma)^{2}+4 \alpha \gamma}}\right)\right. \\
& \times\left(-1-\frac{-1+\alpha+3 \gamma}{\sqrt{(1-\alpha-\gamma)^{2}+4 \alpha \gamma}}\right)+\left(\frac{1-\alpha-\gamma-\sqrt{(1-\alpha-\gamma)^{2}+4 \alpha \gamma}}{2}\right)^{n-1} \\
& \left.\times\left(\frac{-3\left((1-\alpha-\gamma)^{2}+4 \alpha \gamma\right)+(-1+\alpha+3 \gamma)(-1+3 \alpha+\gamma)}{\left((1-\alpha-\gamma)^{2}+4 \alpha \gamma\right)^{3 / 2}}\right)\right] .
\end{aligned}
$$

Thus $D_{1,2} f_{n}(1 / 2,0)=n / 2^{n-3}=D_{2,1} f_{n}(1 / 2,0)$.

Let $H$ denote the Hessian matrix for $f_{n}$ at $(1 / 2,0) . H$ has characteristic values

$$
\lambda_{1}=\frac{n}{2^{n-2}}\left(n-1+\sqrt{(n-1)^{2}+4}\right)
$$

and

$$
\lambda_{2}=\frac{n}{2^{n-2}}\left(n-1-\sqrt{(n-1)^{2}+4}\right) .
$$

Since $\lambda_{2}<0<\lambda_{1}$ then $(1 / 2,0)$ is a saddle point for $f_{n}$. Let $x=\left(\lambda_{2}, 1\right)$ and put $|x|=\sqrt{\lambda_{2}^{2}+1}$. By Taylor's theorem there is a positive number $\delta$ so that if $|x|<\delta$ then there is a number $R(x)$ so that $f_{n}((1 / 2,0)+x)$ is

$$
\frac{1}{0 !} f_{n}(1 / 2,0)+\frac{1}{1 !} \sum_{k=1}^{2}(x)_{k} D_{k} f_{n}(1 / 2,0)+\frac{1}{2 !} \sum_{i, j=1}^{2}(x)_{i}(x)_{j} D_{i, j} f_{n}(1 / 2,0)+R(x)
$$

and therefore, since $(1 / 2,0)$ is a critical point for $f_{n}$, and since $H x^{T}=\lambda_{2} x^{T}$, then

$$
f_{n}((1 / 2,0)+x)=f_{n}(1 / 2,0)+\lambda_{2}|x|^{2}+R(x) .
$$

Since $\lambda_{2}<0$ then there is a positive number $\omega<\delta$ such that if $|x|<\omega$ then $\lambda_{2}|x|^{2}+R(x)<0$, and therefore $f_{n}((1 / 2,0)+x)<f_{n}(1 / 2,0)$. Let $q=(1 / 2,0)+\omega|x|^{-1} x$, observe that $q \in S$ and that $f_{n}(q)<f_{n}(1 / 2,0)$.

THEOREM 5. If $n \in\{3,4\}$ then $f_{n}$ is minimum, uniquely, at $(1 / 3,1 / 3)$.

Proof. In [1] Marcus and Newman proved the van der Waerden 
conjecture true in case $n=3$, and hence this theorem is also true in this case. Let $(\alpha, \gamma)$ be a point of $S$ at which $f_{4}$ is minimum. Observe that $f_{4}(\alpha, \gamma)$ is

$$
\begin{aligned}
& 2 \alpha^{4}-4 \alpha^{3}+6 \alpha^{2}-4 \alpha+2 \gamma^{4}+6 \gamma^{2}-4 \gamma-20 \gamma^{2} \\
& +8 \alpha \gamma^{3}+16 \alpha^{2} \gamma^{2}+8 \alpha^{3} \gamma-20 \alpha^{2} \gamma+16 \alpha \gamma+1,
\end{aligned}
$$

that $D_{1} f_{4}(\alpha, \gamma)$ is

$$
8 \alpha^{3}-12 \alpha^{2}+12 \alpha-4-20 \gamma^{2}+8 \gamma^{3}+32 \alpha \gamma^{2}+24 \alpha^{2} \gamma-40 \alpha \gamma+16 \gamma,
$$

and that $D_{2} f_{4}(\alpha, \gamma)$ is

$$
8 \gamma^{3}-12 \gamma^{2}+12 \gamma-4-40 \alpha \gamma+24 \alpha \gamma^{2}+32 \alpha^{2} \gamma+8 \alpha^{3}-20 \alpha^{2}+16 \alpha .
$$

By Theorem 4, $(\alpha, \gamma)$ is not on the boundary of $S$ and so $D_{1} f_{4}(\alpha, \gamma)=$ $0=D_{2} f_{4}(\alpha, \gamma)$. Thus $D_{1} f_{4}(\alpha, \gamma)-D_{2} f_{4}(\alpha, \gamma)=0$ and therefore

$$
(\alpha-\gamma)(2(\alpha+\gamma)-1-2 \alpha \gamma)=0 \text {. }
$$

Since $D_{1} f_{4}(\alpha, \alpha)=(\alpha-1 / 3)\left(18 \alpha^{2}-12 \alpha+3\right)$ then the only critical point on the diagonal of $S$ is $(1 / 3,1 / 3)$. Suppose

$$
f_{4}(\alpha, \gamma)<f_{4}\left(\frac{1}{3}, \frac{1}{3}\right)
$$

and observe from (1) that

$$
2(\alpha+\gamma)-1-2 \alpha \gamma=0 .
$$

Let $\beta=1-\alpha-\gamma$. It follows from (3) that $\beta^{2}=\alpha^{2}+\gamma^{2}$ and from (2) and (3) that

$$
f_{4}(\alpha, \gamma)=\beta^{4}+2 \beta^{2}(2 \alpha \gamma)+\left(\alpha^{2}+\gamma^{2}\right)^{2}=2 \beta^{2}(1-\beta)^{2}<\frac{1}{9} .
$$

Hence $\beta(1-\beta)<1 / 3 \sqrt{2}$ and therefore

$$
\text { either } \beta<\frac{1-\sqrt{1-\frac{2 \sqrt{2}}{3}}}{2} \text { or } \beta>\frac{1+\sqrt{1-\frac{2 \sqrt{2}}{3}}}{2} \text {. }
$$

It also follows from (3) that $2 \gamma^{2}-2(1-\beta) \gamma+1-2 \beta=0$ and therefore, since $\gamma$ is a real number, then

$$
\beta \geqq \sqrt{2}-1 \text {. }
$$

Finally, (3) implies that $1-2 \beta-2 \alpha \gamma=0$, and therefore since $\alpha \gamma \geqq 0$, then

$$
3 \leqq 1 / 2
$$


Inequalities (4), (5) and (6) constitute a contradiction.

I would like to thank Professor Sinkhorn for his assistance and suggestions in the preparation of this paper.

\section{REFERENCES}

1. M. Marcus and M. Newman, On the minimum of the permanent of a doubly stochastic matrix, Duke Math. J., 26 (1959), 61-72.

2. H. Minc, On permanents of circulants, Pacific J. Math., 42 (1972), 477-484.

Received July 3, 1973.

Missouri Southern State College

JOPLIN, MO 64801 



\section{PACIFIC JOURNAL OF MATHEMATICS}

\section{EDITORS}

DONALD BABBITT (Managing Editor)

University of Galifornia

Los Angeles, California 90024

Hugo RossI

University of Utah

Salt Lake City, UT 84112

C. C. MOORE AND ANDREW OGG

University of California

Berkeley, CA 94720
J. DugundJI

Department of Mathematics University of Southern California Los Angeles, California 90007

R. FinN and J. Milgram Stanford University Stanford, California 94305

\section{ASSOCIATE EDITORS}

R. ARENS

E. F. BeCKenbaCh

B. H. NEUManN

F. WOLF

K. YosHIDA

\section{SUPPORTING INSTITUTIONS}

UNIVERSITY OF ARIZONA

UNIVERSITY OF BRITISH COLUMBIA

CALIFORNIA INSTITUTE OF TECHNOLOGY

UNIVERSITY OF CALIFORNIA

MONTANA STATE UNIVERSITY

UNIVERSITY OF NEVADA, RENO

NEW MEXICO STATE UNIVERSITY

OREGON STATE UNIVERSITY
UNIVERSITY OF OREGON

UNIVERSITY OF SOUTHERN CALIFONIA

STANFORD UNIVERSITY

UNIVERSITY OF HAWAII

UNIVERSITY OF TOKYO

UNIVERSITY OF UTAH

WASHINGTON STATE UNIVERSITY

UNIVERSITY OF WASHINGTON 


\section{Pacific Journal of Mathematics}

\section{Vol. 95, No. $1 \quad$ September, 1981}

John Allen Beachy and William David Blair, On rings with bounded

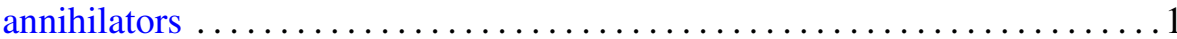

Douglas S. Bridges, A constructive look at positive linear functionals on

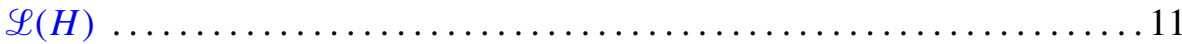

Muneo Chō and Makoto Takaguchi, Boundary points of joint numerical ranges

W. J. Cramer and William O. Ray, Solvability of nonlinear operator equations

Lester Eli Dubins and Gideon Schwarz, Equidiscontinuity of

Borsuk-Ulam functions

Maria Fragoulopoulou, Spaces of representations and enveloping 1.m.c.

*-algebras

Robert F. Geitz and J. Jerry Uhl, Jr., Vector-valued functions as families of scalar-valued functions

Ross Geoghegan, The homomorphism on fundamental group induced by a homotopy idempotent having essential fixed points

Ross Geoghegan, Splitting homotopy idempotents which have essential fixed points

Paul Jacob Koosis, Entire functions of exponential type as multipliers for

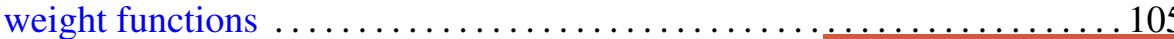

David London, Monotonicity of permanents of certain doubly stochastic matrices

Howard J. Marcum, Two results on cofibers

Giancarlo Mauceri, Zonal multipliers on the Heisenberg group

Edward Wilfred Odell, Jr. and Y. Sternfeld, A fixed point theorem in $c_{0} \quad \ldots 161$

Bernt Karsten Oksendal, Brownian motion and sets of harmonic measure zero

Andrew Douglas Pollington, The Hausdorff dimension of a set of normal numbers

Joe Repka, Base change lifting and Galois invariance ...

Gerald Suchan, Concerning the minimum of permanents on doubly stochastic circulants

Jun-ichi Tanaka, On isometries of Hardy spaces on compact abelian groups

Aaron R. Todd, Quasiregular, pseudocomplete, and Baire spaces 